\title{
COMPARATIVE STUDY BETWEEN OPEN AND PERCUTANEOUS REPAIR FOR ACUTE ACHILLES TENDON RUPTURE
}

\author{
Hossam Hosny ${ }^{(*)}$, Mostafaa Hemaid, Abd-El-Rahman Hafez \\ Orthopaedic dept, Faculty of Medicine, Sohag University, Sohag, Egypt \\ *E-mail: hosam09@yahoo.com
}

Received 12/12/2020

Accepted 4/3/2021

\begin{abstract}
There is controversy between conservative and operative treatment of acute achilles tendon rupture. Operative treatment of achilles tendon rupture can be done through both percutaneous and open technique. Open repair of achilles tendon rupture was associated with higher incidence of wound complications while percutaneous repair was associated with higher incidence of tendonre-rupture rate and sural nerve injury. In this study, we aim to assess the functional outcomes of both open and percutaneous repair of acute achilles tendon rupture and the incidence of complications in both repair types. Twenty patients with acute achilles tendon rupture were retrospectively reviewed and evaluated. Ten patients were treated through percutaneous repair and ten patients were treated through open repair. The patients were followed up for at least one year and evaluated at the last follow up visit using Achilles Tendon Rupture Score (ATRS). Complications for each group were recorded. Twenty patients were included in our study. Ten patients were subjected to percutaneous repair and ten patients were subjected to open repair. There were two case of wound infection and one case of delayed skin healing in patients treated with open repair. The Achilles tendon Rupture Score (ATRS) yielded nearly similar results for both groups at the final follow up. There is no difference between open and percutaneous repair of achilles tendon rupture at a long-term follow up. Both groups had equal functional outcomes however the percutaneous repair of achilles tendon was associated with less wound complications than open repair
\end{abstract}

Keyword: Achilles tendon, Open versus percutaneous repair, Complications of achilles tendon repair Achilles rupture, percutaneous repair, minimally invasive

\section{Introduction}

The Achilles tendon is the primary plantar flexor of the ankle joint and it is considered the strongest tendon in the body [1]. The achilles tendon rupture most commonly occurs in the third to fourth decade of life specifically in males during sports activities
$[2,3]$. Controversy is existed between conservative and operative treatment of acute achilles tendon rupture [4-6]. However operative treatment was proved to have the best functional results and more patient satisfaction [4,7-9]. Non operative mana- 
gement is associated with re-rupture rates of $13 \%$ to $33 \%[4,6]$. The operative treatment has the benefit of early mobilization, lower re-rupture rates, and earlier return to sports practice and daily activities [810] but wound complications rate are more commonly occur with a percent varies from $0 \%$ to $22 \%[11,12]$. Open and percutaneous repair do exist for managing patients who had acute achilles tendon rupture. Amlang et al. introduced the percutaneous repair technique of achilles tendon rupture [13]. The blood supply of achilles tendon comes from three sources. Distally, the tendon had a blood supply from the peri-osteum surrounding the calcaneal bone. The middle part takes blood supply from the paratenon which is considered the main blood supply while the proximal part had a blood supply from the myotendinous junction. The achilles tendon is divided into three zones according this pattern of blood vascularity. Zone I is less than $3 \mathrm{~cm}$ from the insertion of the tendon in the calcaneus. Zone II is about 3-6 $\mathrm{cm}$ from tendon insertion in the calcaneus while zone III is more than $6 \mathrm{~cm}$ from tendon insertion in the calcaneus. The commonest injury of achilles tendon occurs in Zone II [14]. The main aim of repairing acute achilles tendo nrupture is to restore the anatomical length of the triceps surae through re-approximation of the torn ends of the achilles tendon to restore the normal biomechanics around the ankle joint. This can be accomplished through preserving the blood supply of the achilles tendon, avoid damage to the tendon substance to maintain its functional properties and protecting the surrounding vital structures. In our study, we aimed to assess the functional outcomes of both open and percutaneous repair of acute achilles tendon rupture and the incidence of complications in both repair types.

\section{Patients and Methods}

Twenty patients with surgically treated acute achilles tendon rupture were retrospectively reviewed and functionally evaluated. The study was conducted between January 2017 and January 2019. Our Intuitional Review Board approved the study.

\subsection{Inclusion criteria}

Patients with acute rupture of the acute achilles tendon rupture whether open or closed injury.

\subsection{Exclusion criteria}

1) Patients with extensive soft tissue damage and lacerated wounds.

2) Patients with pathological achilles tendon rupture as post steroid injection. All patients were evaluated clinically and radiologically. Clinically, the patients were unable to actively planter-flex the ankle joint and there was a palpable a gap palpable at the distal part of the tendon. Thompson test through Calfsqueezing was positive in all patients. Plain radiographs including anteroposterior and lateral views of the ankle were done to exclude associated fractures. All necessary investigations had bone done before surgery.

\subsection{Surgical technique}

Prone position was used in all patients. The patient's leg can be elevated using a pillow which placed under patient's leg. This position facilitates good adaptation of the tendon parts and prevents excessive plantar flexion to limit over tightening. Antibiotics (first generation cephalosporin) had been administrated half an hour before elevating the tourniquet which was inflated after full exsanguination of the limb. Saline wash was mandatory especially in cases with open wounds.

\subsection{For percutaneous repair}

Three slit shaped incisions were done medial and lateral to the proximal portion 
of the tendon and two slit shaped incision were done medial and lateral to the distal portion. Absorbable sutures (Vicryl No. 2 Ethicon) were used with Mayo needle. The proximal portion was captured by a suture passed transverse the $1^{\text {st }}$ proximal slits, then suture was crossed to the $2^{\text {nd }}$ proximal slits and to the $3^{\text {rd }}$ proximal slits. Sutures were crisscrossed through the gap to
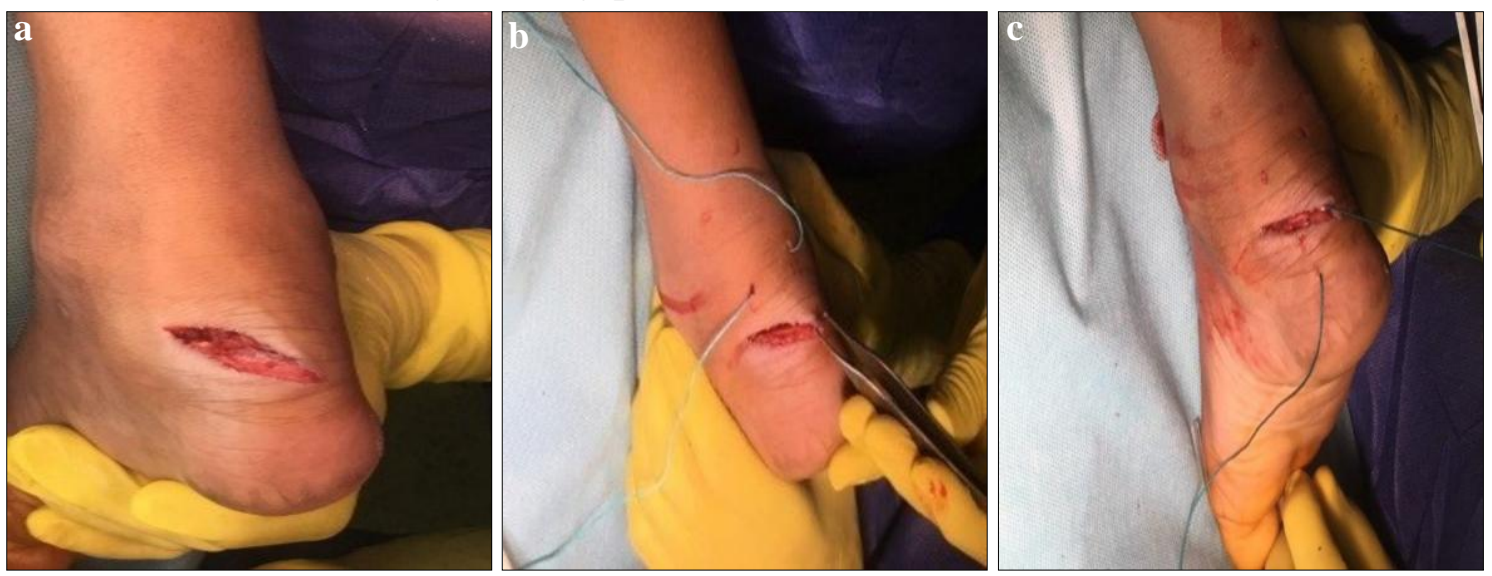

Figure (1) shows a. intraoperative photo showing open rupture of achilles tendon and repair through percutaneous technique, b. the proximal part of the tendon is captured by sutures through the three slit shaped incisions, c. the sutures were crisscrossed through the wound gap to reattach the proximal and distal parts of tendon.

\subsection{For open repair}

Posteromedial approach was used to expose the injured part of the tendon. The paratenon was carefully incised in a longitudinal direction. We had to protect the neurovascular bundle which is located medially. After trimming the ruptured muscle fibers. The tendon was repaired using absorbable
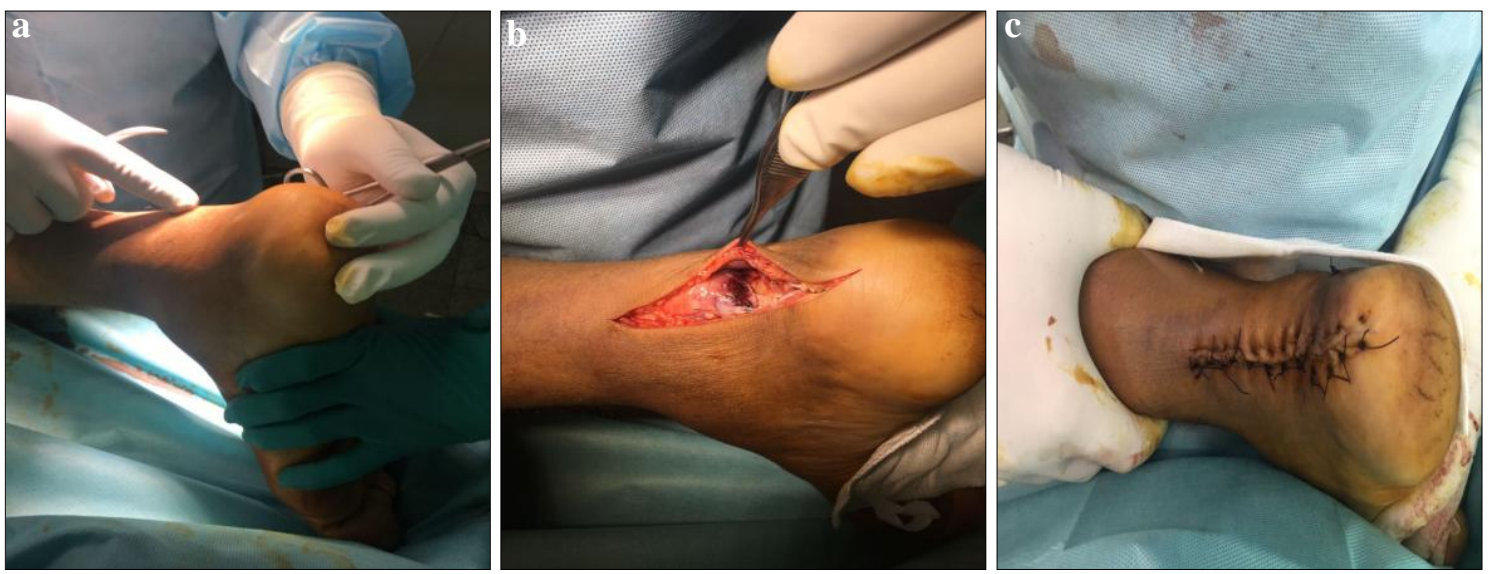

Figure (2) shows a. pre-operative photo showing closed rupture of achilles tendon and repair through open technique, b. intraoperative photo showing both ends of achilles tendon through posteromedial approach, c. intraoperative photo showing skin closure after open repair of achilles tendon. 


\subsection{Postoperative treatment}

Immobilization in a below knee cast for four weeks with the foot in maximum planter flexion was done for all patients. Stitches were removed after14 day. Physiotherapy started after removal of the cast at four weeks. All patients followed a standardized rehabilitation protocol. All patients were followed up for at least 12 months. Evaluation of the patients regarding ankle movements, pain and associated complications was done at 4 weeks, 3, 6 and 12 months. At the last visit, all patients were functionally evaluated according to the achilles tendon rupture score (ATRS) [15].

\section{Results}

Twenty patients were included in our study. 10 patients were subjected to percutaneous repair and 10 patients were subjected to open repair. All patients were followed up for at least one year. Age range from 10 to 60 years. Mechanism of injury was sharp objects in 15 patients, ruptureeachilles tendon while practicing sports in 3 patients and 2 patients had injury due to falling from height. 17 patients were male.
The right foot was affected in 12 cases. 17 case had open acute achilles tendon rupture while 3 case had closed acute achilles tendon rupture, tab. (1). The mean time for patients' return to work was 7 weeks in cases who underwent percutaneous achilles tendon repair group and 9 weeks for patients who had open achilles tendon repair group. At the last follow up, all patients had returned to their normal daily activities with no morbidity. Wound infection occurred in two cases while delayed skin healing occurred in one case in patients who treated with open repair and they were treated with antibiotics, continuous dressing and complete wound healing had been occurred with no residual complications apart from a big skin scar. These complications were not experienced in patients with percutaneous repair. Other complications as skin necrosis, wound fistula, sural nerve injury and tendon rerupture had not been experienced throughout the study in both groups. All patients were subjected to Achilles Tendon Rupture Score (ATRS) at the final follow up. The score (ATRS) yielded nearly similar results for both groups

Table (1) showing results of our study

\begin{tabular}{|c|c|c|c|c|c|c|c|c|c|}
\hline & Age & Sex & Side & $\begin{array}{l}\text { Open or closed } \\
\text { achilles rupture }\end{array}$ & Mechanism of injury & $\begin{array}{l}\text { Mean Period to } \\
\text { return to work }\end{array}$ & $\begin{array}{l}\text { Mean follow } \\
\text { up period }\end{array}$ & $\begin{array}{c}\text { Mean Achilles } \\
\text { tendon rupture score }\end{array}$ & Complication \\
\hline 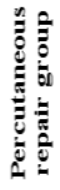 & 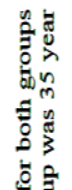 & $\begin{array}{l}\text { Male (8) } \\
\text { Female (2) }\end{array}$ & $\begin{array}{l}\text { Right side (5) } \\
\text { Left side (5) }\end{array}$ & $\begin{array}{l}\text { Open injury (8) } \\
\text { Closed Injury (2) }\end{array}$ & $\begin{array}{c}\text { 1-Sharp objects (7) } \\
\text { 2-Practicing spots (2) } \\
\text { 2-Falling from height (1) }\end{array}$ & 7 weeks & 15 month & 88 & None \\
\hline 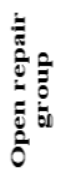 & 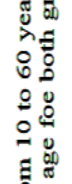 & $\begin{array}{l}\text { Male (9) } \\
\text { Female (1) }\end{array}$ & $\begin{array}{l}\text { Right side (7) } \\
\text { Left side (3) }\end{array}$ & $\begin{array}{l}\text { Open injury (9) } \\
\text { Closed Injury (1) }\end{array}$ & $\begin{array}{c}\text { 1-Sharp objects (8) } \\
\text { 2-Practicing sports (1) } \\
\text { 3-Falling from height (1) }\end{array}$ & 9 weeks & 18 month & 85 & $\begin{array}{l}\text { Two case of } \\
\text { wound infection } \\
\text { and one case of } \\
\text { delayed skin } \\
\text { healing. }\end{array}$ \\
\hline है & 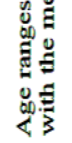 & $\begin{array}{l}\text { Male (17) } \\
\text { Female (3) }\end{array}$ & $\begin{array}{l}\text { Right side(12) } \\
\text { Left side (8) }\end{array}$ & $\begin{array}{l}\text { Open injury (17) } \\
\text { Closed Injury (3) }\end{array}$ & $\begin{array}{l}\text { 1-Sharp objects (15) } \\
\text { 2-Practicing sports (3) } \\
\text { 2-Falling from height (2) }\end{array}$ & & & & \\
\hline
\end{tabular}




\section{Discussion}

The achilles tendon is commonly injured during sports activity or with sharp instruments [16]. Direct trauma (sharp objects) or indirect trauma (during sport practice) are the main mechanisms of acute achilles tendon rupture. Spontaneous rupture may occur in patients with autoimmune diseases, infectious diseases, collagen diseases and inflammatory diseases [14]. The main aim of treatment of acute achilles tendon rupture is to minimize the morbidity, had both acceptable and satisfactory clinical and functional outcomes at the final followup and to prevent the incidence of associated complications. There are multiple open surgical procedures that were used to repair acute achilles tendon rupture but no one is superior. Wound infection, delayed healing, adhesions and skin necrosis are potential complications that may occur with open procedures and more commonly seen in diabetic patients moreover these open procedures may be associated with delayed tendon healing time and this was not suitable for sport practitioners who wish to return earlier to their pre-injury activity level [2,17-19]. Amlang et al [13]. Introduced the technique of percutaneous acute achilles tendon repair to overcome these complications but tendon re rupture and sural nerve injury were a frequent complications [20-22]. Although the percutaneous treatment decreases wound complication rate, the incidence of sural nerve injury rate increases as high as $16.7 \%$ [23,24]. Our objective in this study is to assess the functional outcomes and the incidence of complications in both techniques for achilles tendon repair. We observed that the percutaneous achilles tendon repair group had the advantage of minimally invasive technique with less complications as wound infection, delayed skin healing and rapid return to work and daily activities while the group with open achilles tendon repair was associated with increased incidence of wound complication as infection, delayed skin healing, scar formation and late return to sport and daily activities. These observations were at the short term follow up period (first 6 months) while at the long follow up period (mean one year), both groups had nearly the same functional outcomes with complete satisfaction of all patient of both groups. We did not experienced complications as sural nerve injury or tendon re-rupture throughout our study. Several studies concerned with comparing both open and percutaneous achilles tendon repair do exist in the literature. Cretnik et al [25]. Compared two groups with open and percutaneous achilles tendon repair and his results was similar to our results. They reported that no statistically significant difference in functional outcomes between open surgical repair and percutaneous repair of acute achilles tendon rupture and there were statistically significant major complications were associated with the open achilles tendon repair group. Karabinas et al [26] operated 34 patients with acute Achilles tendon ruptures. $\mathrm{He}$ reported that both percutaneous and open achilles tendon repair had similarly successful clinical and functional outcomes however cosmetic appearance was better in the group of patients treated with percutaneous achilles tendon repair. Gigante et al [27] reported that both methods of repairing acute achilles tendon rupture were safe and effective under the same rehabilitation program. Lim et al. [21] had been operated 66 patients with acute achilles tendon rupture with both percutaneous and 
open repair technique. They observed a high rate of complication in open repair group. There were 7 cases of wound infections and two re-ruptures in the open group compared to 3 cases of wound puckering and one re-rupture in the percutaneous group. Henriquez et al [6] reported that both percutaneous and open achilles tendon repair had a similar function outcome but the percutaneous technique was associated with a better cosmetic appearance, a lower rate of wound complications, and no apparent increase in the risk of re-rupture. Both techniques open and percutaneous repair were effective in treatment of acute achilles tendon rupture however the percutaneous repair was associated with less wound complications than open repair however both techniques had the same functional outcomes at the long-term follow up. One of the limitations of this study is small sample size so we unable to do a statistical analysis.

\section{Conclusion}

There is no difference between percutaneous and open repair of achilles tendon at the long-term follow up. Both groups had equal functional outcomes however the percutaneous repair of achilles tendon was associated with less wound complications than open repair. We advise percutaneous achilles tendon repair as it was associated with less wound complications and better cosmetic appearance compared to open achilles tendon repair.

\section{References}

[1] Järvinen, T., Kannusm P., Maffulli N., et al. (2005). Achilles tendon disorders: Etiology and epidemiology. Foot and Ankle Clinics. 10 (2): 255-266.

[2] Bruggeman, N., Turner, N., Dahm, D., et al. (2004). Wound complications after open Achilles tendon repair: an analysis of risk factors. Clinical Orthopaedics and Related Research ${ }^{\circledR}$. 427: 63-66.

[3] Suchak, A., Bostick, G., Reid, D., et al. (2005). The incidence of Achilles tendon ruptures in Edmonton, Canada. Foot \& ankle int. 26 (11): 932-936.

[4] Kocher, M., Bishop, J., Marshall, R., et al. (2002). Operative versus nonoperative management of acute Achilles tendon rupture: Expected-value decision analysis. The American $J$. of Sports Medicine. 30 (6):783-790.

[5] Maffulli, N. (1999). Current concepts review-rupture of the Achilles tendon. JBJS. 81 (7):1019-1036.

[6] Henríquez, H., Muñoz, R., Carcuro, G., et al. (2012). Is percutaneous repair better than open repair in acute Achilles tendon rupture? Clinical Orthopaedics and Related Research ${ }^{\circledR} .470$ (4): 998-1003.

[7] Assal, M., Jung, M., Stern, R., et al. (2002). Limited open repair of Achilles tendon ruptures: A technique with a new instrument and findings of a prospective multicenter study. JBJS. 84 (2):161-170.

[8] Wong, J., Barrass, V., Maffulli, N. (2002). Quantitative review of operative and nonoperative management of Achilles tendon ruptures. The American J. of Sports Medicine. 30 (4): 565-575.

[9] Rippstein, P., Jung, M., Assal. M. (2002). Surgical repair of acute Achilles tendon rupture using a "mini-open" technique. Foot and Ankle Clinics. 7 (3): 611-619.

[10] Cetti, R., Christensen, S-E., Ejsted, R., et al. (1993). Operative versus nonoperative treatment of Achilles tendon 
rupture: A prospective randomized study and review of the literature. The American J. of Sports Medicine. 21 (6): 791-799.

[11] Bhandari, M., Guyatt, GH., Siddiqui, F., et al. (2002). Treatment of acute Achilles tendon ruptures a systematic overview and metaanalysis. Clinical Orthopaedics and Related Research®. 400: 190-200.

[12] Khan, R., Fick, D., Brammar, T., et al. (2004). Surgical interventions for treating acute Achilles tendon ruptures. Cochrane Database of Systematic Reviews, Doi: 10.1002/14651858.

[13] Amlang, M., Christiani, P., Heinz, P., et al. (2005). Percutaneous technique for Achilles tendon repair with the Dresden instruments. Der Unfallchirurg. 108 (7): 529-536.

[14] Hosny, H., Salama, W., Marzouk, A., (2020). Repair of traumatic avulsed Achilles tendon using double cerclage trans-calcaneal stainless steel sutures: Prospective study and review of literature. Injury. 51 (8): 1887-1892.

[15] Nilsson-Helander, K., Thomeé, R., Grävare-Silbernagel, K., et al. (2007). The Achilles tendon total rupture score (ATRS) development and validation. The American J. of Sports Medicine. 35 (3): 421-426.

[16] Möller, A., Åström, M., Westlin, NE. (1996). Increasing incidence of Achilles tendon rupture. Acta orthopaedica Scandinavica. 67 (5): 479-481.

[17] Chan, K., Lui, T., Chan, L. (2009). Endoscopic-assisted repair of acute Achilles tendon rupture with Krackow suture: An anatomic study. Foot and Ankle Surgery. 15 (4): 183-186.

[18] Metz, R., van der Heijden, GJ., Verle- isdonk, E-JM, et al. (2009). Recovery of calf muscle strength following acute achilles tendon rupture treatment: A comparison between minimally invasive surgery and conservative treatment. Foot \& Ankle specialist. 2 (5): 219-226.

[19] Gillies, H., Chalmers, J. (1970). The management of fresh ruptures of the tendo achillis. JBJS. 52 (2): 337-343.

[20] Haji, A., Sahai, A., Symes, A. (2004). Percutaneous versus open tendo achillis repair. Foot \& Ankle Int. 25 (4): 215-218.

[21] Lim, J., Dalai, R., Waseem, M. (2001). Percutaneous vs. open repair of the ruptured Achilles tendon - a prospective randomized controlled study. Foot \& Ankle Int. 22 (7): 559-568.

[22] Maes, R., Copin, G., Averous, C. (2006) Is percutaneous repair of the Achilles tendon a safe technique? A study of 124 cases. Acta Orthopaedica Belgica.;72(2):179.

[23] Buchgraber, A., Pässler, HH. (1997). Percutaneous repair of Achilles tendon rupture: Immobilization versus functional postoperative treatment. Clinical Orthopaedics and Rrelated Research. (341): 113-122.

[24] Khan, R., Fick, D., Keogh, A, et al. (2005). Treatment of acute Achilles tendon ruptures: A meta-analysis of randomized, controlled trials. JBJS. 87 (10): 2202-2210.

[25] Čretnik, A., Kosanović, M., Smrkolj, V. (2005). Percutaneous versus open repair of the ruptured Achilles tendon: a comparative study. The American J. of Sports Medicine. 33 (9):13691379.

[26] Karabinas, P., Benetos, I., Lamprop- 
oulou-Adamidou, K., et al. (2014) Percutaneous versus open repair of acute Achilles tendon ruptures. European J. of Orthopaedic Surgery \& Traumatology. 24 (4): 607-613.

[27] Gigante, A., Moschini, A., Verdenell, et al. (2008). Open versus percutaneous repair in the treatment of acute Achilles tendon rupture: A randomized prospective study. Knee Surgery, Sports Traumatology, Arthroscopy. 16 (2): 204-209. 